\title{
In Situ Calibration Algorithms for Environmental Sensor Networks: a Review
}

\author{
Florentin Delaine, Bérengère Lebental and Hervé Rivano
}

\begin{abstract}
The recent developments in both nanotechnologies and wireless technologies has enabled the rise of small, low cost and energy efficient environmental sensing devices. Many projects involving dense sensor networks deployments have followed, in particular within the Smart City trend. If such deployments are now within economical and technical reach, their maintenance and reliability remain however a challenge. In particular, reaching, then maintaining, the targeted quality of measurement throughout deployment duration is an important issue. Indeed, factory calibration is too expensive for systematic application to low-cost sensors and as these sensors are usually prone to drifting because of premature aging. In addition, there are concerns about the applicability of factory calibration to field conditions. These challenges have fostered many researches on in situ calibration. In situ means that the sensors are calibrated without removing them from their deployment location, preferably without physical intervention, often leveraging their communication capabilities. It is a critical challenge for the economical sustainability of networks with large scale deployments.

In this paper, we focus on in situ calibration methods for environmental sensor networks. We propose a taxonomy of the methodologies in the literature. Our classification relies on both the architecture of the network of sensors and the algorithmic principles of the calibration methods. This review allow us to identify and discuss two main challenges: how to improve the performance evaluation of such methods and how to enable a quantified comparison of these strategies?
\end{abstract}

Index Terms-Sensor networks, calibration, algorithms

\section{INTRODUCTION}

$\mathbf{E}$ NVIRONMENTAL sensors are measuring instruments designed to measure ambient quantities such as temperature, relative humidity, noise, pressure, wind speed, wind direction, chemical components concentrations and so on. Fostered by the emergence of the Internet of Things (IoT) and of low-cost sensing devices, the interest for these devices has been growing for the past decades [1].

Depending on the target area to monitor and on the spatial variability of the measurand, hundreds of devices may be required, as shown for instance by [2] and [3] in the field of air quality monitoring. The cost of sensors then becomes a major factor, which explains the interest for low-cost sensors [4]. These technologies are still emerging and must face several issues, one of them being the improvement of data quality [1] [5] [6] [7] [8].

Florentin Delaine and Bérengère Lebental are with Efficacity, F-77420 Champs-sur-Marne, France, Université Paris-Est, IFSTTAR, COSYS, F77447 Marne-la-Vallée, France and Laboratoire de Physique des Interfaces et Couches Minces (LPICM), École Polytechnique, CNRS, Université ParisSaclay, F-91128 Palaiseau, France.

Hervé Rivano is with Université de Lyon, INSA Lyon, Inria, CITI, F-69621, Villeurbanne, France.

Corresponding author: Florentin Delaine (florentin.delaine@gmail.com)
More precisely, it is often observed that the sensing quality of low cost devices decay with time, even under regular operating conditions [9]. In particular, the calibration relationship may change, hence the need for frequent evaluation. In metrological terms [10], calibration consists in deriving, under specified conditions, the relationship between the indication of the instrument (its raw output) and the measurand (the quantity intended to be measured). This is a quite well mastered operation when performed in dedicated laboratory facilities where most parameters can be controlled and almost perfectly known. However, in such conditions recalibration of a deployment of sensors means dismounting and shipping the whole network from the field to a calibration facility and re-deploy it afterward. It is not technically and economically sustainable for dense deployments of low cost devices.

Consequently, calibration procedures suitable for sensor placed in field conditions have been widely investigated in the past two decades. This is all the more relevant as the validity of laboratory calibrations is often questioned in the field [11] [12]. Furthermore, a significant part of commercial sensor technologies are sold without initial individual calibration (see for instance [13]) to reduce cost.

In this paper, we review the literature on in situ calibration methods for environmental sensor networks. In situ methods, sometimes called field, in place, remote or online calibration instead, enable to calibrate measuring instruments of a network while leaving them deployed in the field, preferably without physical intervention. The literature on the subject studies under which hypotheses, in which manner and with which performance the measured values from a sensor network may be exploited to improve the measurement accuracy of the whole network through calibration. In a recent survey, Maag et al. focus on the use case of air pollution monitoring, addressing operational concerns regarding to calibration [14].

In the present paper, we propose a review of in situ calibration strategies with a different scope: we address all environmental sensor networks, regardless of the monitored phenomenon. We also classify the literature with regard to the underlying algorithmic approaches. More precisely, we propose a synthetic taxonomy of the large variety of different techniques reported under different terms in the literature, such as "blind calibration", "multi-hop calibration", "macro calibration" and so on. Additionally, we consider the architecture of the sensor network on which depends the relevance of each strategy.

The review is organized as follows. Section $\Pi$ defines the terms used and details the scope of our literature review. A taxonomy is then proposed for the classification of existing 
techniques of in situ calibration for environmental sensors networks in Section III. In Section IV] various contributions are analyzed and positioned regarding the previous categories. Section $\mathrm{V}$ is dedicated to a discussion on how to bring to a next level the performance evaluation of in situ calibration methods and their quantified comparison. Finally, Section VI gives a conclusion.

\section{DEFINITIONS AND SCOPE}

An environmental sensor network is a set of measuring systems [10] spatially deployed in order to periodically measure one or more quantities in an environment. Measuring systems are also called nodes. Each may be composed of one or more measuring instrument according to the definition of a measuring system. A node may be static or mobile. The set of nodes forms a (most probably wireless) network. It can be either meshed, with device to device communications, or a collection of stars centered on gateways. For a given measurand, instruments which are known to be more accurate than the others of the network are called reference instruments.

The terminology used in this paper is inspired by the field of dependability [15] [16]. In particular, recalibration methods are contributing to the reliability of the measuring instruments. Reliability is indeed the ability to continuously deliver a correct service i.e., accurate values in our settings.

There are other considerations that could matter and interact with calibration, such as integrity, security or even privacy, in particular when considering crowdsensing platforms involving citizens [17]. As a matter of fact, their integration at an early step of the design of the sensor networks is crucial with respect to the system architecture. The way these concepts are implemented may have a significant impact on the effectiveness of some calibration methods. We however do not consider them in the scope of this survey because our classification relies on the network architecture and the algorithmic principles underlying the calibration method, as detailed in the following section. This viewpoint is hardly influenced by system issues unless practical implementation details are considered.

\section{ClassificATION}

The following subsections introduce our taxonomy for the classification of in situ calibration strategies for sensor networks (SN).

The classification is independent from the kind of mesurand: the groups of categories described are relevant for any environmental phenomenon.

We consider network architecture characteristics, namely the nature of instruments and their potential mobility, and the algorithmic principles of the calibration techniques, namely the mathematical structure of the calibration relationship and to which point the algorithm can be distributed.

Each subsection represents a primary level group of categories that may have others nested. Categories for each group are in bold font.

\section{A. Use of reference instruments}

One of the first criteria of classification is how the calibration method assumes the presence of reference instrument within the network.

The calibration of measuring instruments using a sufficient number of reference measurement instruments is called reference-based calibration. It means that the network is composed of both reference and non-reference instruments and that all the non-reference instruments can be calibrated using at least one reference instrument. The approach postulates the existence of a calibration relationship between each nonreference instrument and at least one reference instrument because there are close enough for instance.

The calibration of measuring instruments in the absence of reference values is called blind calibration. It means that the network is composed of only non-reference instruments. These various methods may or may not assume the existence of a correlation between the instrument outputs.

The hybrid situation is called partially blind calibration. In this setting, the network may gather both reference and nonreference instruments, but a reference based calibration is not achievable, e.g when some of the non-reference instruments can never be compared to a reference instrument. It also capture cases where some non-reference instruments are considered good enough to approximate a reference instruments compared to the others.

\section{B. Mobility of the instruments}

The second significant aspect of the network architecture is the potential mobility of nodes.

A first category of methods addresses network with exclusively static nodes. A second one addresses networks with exclusively mobile nodes. The corresponding methods rely often strongly on the mobility of the nodes to achieve calibration. A last group of methods addresses heterogeneous networks with both mobile and static nodes. In such cases the mobility of the nodes is not systematically exploited in the calibration strategy.

\section{Calibration relationships}

The purpose of calibration is to establish a mathematical relationship between the indications of the instrument and the measurand. This category is first based on the number of kind of quantities as input variables in the relationship: the measurand, the indications, the influence quantities, and so on. In terms of algorithmic principles of the calibration methods, it implies the variety and quantity of data to exchange as well as the computational effort that are necessary to achieve a target accuracy.

The most straightforward relationships are called monokind variables without time. They only take a single quantity as input variable and do not depend on time.

The second category of relationships gathers the ones that have mono-kind variables with time. It accounts for a relationship with mono-kind variables which is influenced by time, for instance in case of sensor drifting due to aging [18]. 
The relationships with multiple-kind variables without time account for two or more quantities as variables but remain independent from time. These models are mainly used to include the effect of influence quantities in the calibration relationship. In these case, the networks include instruments measuring the influence quantities. They are not systematically reference instruments and therefore their calibration may also be included in the calibration strategy.

Finally, this last approach may be extended into relationships wind multiple-kind variables with time when appropriate.

For each of these categories, sub-categories can be defined based on the kind of mathematical expression used for the calibration relationship. Popular examples are the following:

- polynomial with constant coefficients [19],

- gain-phase [20],

- variable offset [21],

- neural network [22].

\section{Instrument grouping strategies}

While the previous categories are mostly driven by operational constraints (deployment strategy, properties of the measurand and of the selected sensors), the present paragraph considers the number of nodes involved in each calibration step and to which point the algorithm can be distributed.

A first approach is pairwise calibration. Two instruments are used, one providing standard values for the other. It is classically applied between a reference instrument (or approximation of reference) and each of the nodes related to it. It can be a distributed or even localized algorithm.

A macro calibration strategy consists in calibrating the network as a whole. Even if they exist, the node-to-node relationships are not exploited directly. A centralized algorithm might be necessary with this grouping strategy.

Group calibration is an intermediate approach consisting in carrying calibration operation among groups of measuring instruments among the whole network. In this case, the criteria defining these groups become essential. This approach may be used when pairwise calibration induces significant error, while macro calibration is not fine-tuned enough. This category notably includes strategies where groups are composed of instruments measuring additional quantities besides the main target quantity. These additional quantities are often included as influence quantities in the calibration relationship. These algorithms can be at least partially distributed e.g., computation concentrated on an elected group leader, or fully distributed at the cost of messages broadcasting.

\section{REVIEW OF THE LITERATURE BASED ON THIS CLASSIFICATION}

An application of the classification is provided here with highlights on the existing literature. Table I sorts a large number of in situ calibration studies according to this classification. Some rows refer to multiple papers as they are related somehow (same technique or same authors) and consist in developments of the same initial paper. The current section focuses on a description of the methods. The topic of performance comparison between methods is addressed in the next section. The addressed measurands cover a wide range of environmental quantities: temperature [19], pressure [23], noise [24], air pollutants [25], light [26]... Most of the reported studies have generic approaches that can be transposed to other measurands.

\section{A. Overview}

Regarding to pairwise strategies, relatively few papers address methodological issues related to reference-based pairwise strategies, as this approach is the closest to a "traditional" calibration approach with measurement standards and features less challenges. Partially blind and blind pairwise calibration methods (often focusing on mobile nodes) are more complex as they require to define calibration relationships not only between reference and non-reference nodes, but also between non-reference nodes only. This translates into error propagation issues.

Macro calibration approaches were initially developed to address the absence of reference instruments in a network and thus are mostly blind or partially-blind. In the absence of reference, there is a strong challenge in defining valid calibration relationships based on non-reference sensors data, which explains the strong interest for these methods.

Group strategies have been generating strong interests as they appear to outperform both pairwise and macro strategies with or without reference instruments.

Most methods are based on relationships with mono-kind variables without time and with a linear expression, but more complex models are progressively appearing to better address the complexity of environmental sensing.

Likewise, while most work initially focused on static networks, there are now many interests for mobile nodes as they allow for physical rendez-vous between nodes. Henceforth, calibration methods are less impacted by the physical variability of the phenomena.

Finally, an underlying question addressed is the ability to distribute the computation of calibration relationships [24] [27] [28] [29] [30] [31] [32]. The topic is of strong interest when considering privacy preservation issues [17]. The capability to decentralize is linked to the grouping strategy: pairwise and group strategies foster more naturally decentralized computation, under the condition that the nodes are capable of individual procession and of bidirectional communication. On the contrary, macro-calibration strategies tend to be centralized, except when the characteristics of the parameter identification methods allow for partially or fully decentralized computation. However, while distributed computing impacts the computational performance of algorithm, there is no report on how it affects calibration performance so far.

\section{B. Mobile and static nodes}

Static networks are more frequently studied than mobile ones. A wide range of solutions is now available to calibrate them. However, these calibration methods usually require a high spatial density of nodes to overcome the spatial variability 
of the phenomena, which is not always viable technically or economically. The availability of mobile nodes could alleviate this constraint, as calibration operations exploit physical rendez-vous between nodes. In turn, the methods based on this principle are challenged when the rendez-vous frequency is too low compared to the speed of degradation of the measurement accuracy [33]. In such cases, the addition of a few reference nodes seems to yield satisfying results [25] [34]. Moreover, a challenge of mobile sensors is that they face rapid transients. To address this, methods initially developed for static networks appear promising, such as the work of De Vito et al. 222 [35] which uses dynamic and nonlinear supervised machine learning tools.

\section{Calibration relationships}

Most reported relationships are of mono-kind variables without time type and based on linear expressions. Nevertheless, there is a rising interest for models with multiplekind variables, which stems from the observation that there are indeed significant influence quantities for various environmental measurands, notably air pollutant concentrations. It often depends on the technology of the sensors used [36] [37] [38] [39] [40]. Such relationships gave very interesting results compared to simpler relationship models:

- for reference-based group calibration in [41] [42] and [43]

- for partially blind group calibration strategies in [38] [44] [45] [46], including with time-sensitive models in [29] and [44]

- for blind strategies, either pairwise or group based, in [35] [47] [48] [49] [50].

On the contrary, relationships with multiple-kind variables were shown to be unnecessary in [51] and in [52] where the control of the operating temperature of the device was sufficient to perform a pairwise calibration without being influenced by this quantity.

In general, time-dependent approaches are used to address drift issues. Drift is often modeled as an additive random variable with a given probability distribution [28] [53], so that drift-compensation translates as an offset correction.

\section{Pairwise strategies}

a) Reference-based pairwise: Relatively few papers address methodological issues related to reference-based pairwise strategies, as this approach is the closest to a "traditional" calibration approach with measurement standards. Primarily, reference instruments may be directly co-located in the field with non-reference instruments to achieve their calibration [38] [41] [42] [54] [55].

However, more automated strategies are expected, requiring less the co-location of instruments. Nevertheless, even in the simple case of a relatively dense $\mathrm{SN}$, the measurand may spatially vary too much in general to relate a reference instrument at a given location to an instrument at another location for calibration purposes. As an elementary solution to this, Moltchanov et al. [56] proposed to carry out calibration against the reference node using only the data collected during a specific time span based on the postulate that the phenomenon varies less during this time span. This was an idea previously developed by Tsujita et al. [57] including weather conditions that were also used to corrected the measured values but not with a reference-based approach.

b) Partially blind pairwise: Partially blind pairwise calibration focuses mostly on mobile nodes. Tsujita et al. [58 tackled it first for mobile nodes by proposing that the device to calibrate should display either the value of a reference node that is close enough, or the average measurements between colocated nodes if no reference node is available. A calibration parameter is tuned with these values to correct measurements between rendez-vous.

Y. Xiang et al. [28] later proposed another method. They also distinguish calibration based on the values of a reference instrument and on the values of a non-reference instrument. Their originality relies in the correction of the values that is performed with an estimator of the drift error of the node. This error is recalculated at each calibration by minimizing its variance according to a linear combination of the values of the sensors involved in the calibration process.

Hasenfratz et al. [25] addressed by various methods the case of calibration for mobile devices against reference instruments or not. They notably provided dedicated extensions for the case where some devices rarely encounter reference instruments. They also demonstrated a linear dependency between the measurement error and the number of intermediary calibrations between a given node and the reference node it is calibrated against. In [33] and [59], Saukh et al. proposed solutions to this issue of error accumulation by working on the occurrence of rendez-vous between nodes, in view of maximizing the opportunities of calibration. An alternative idea was developed by $\mathrm{Fu}$ et al. [60] who proposed the optimization of the deployment of reference instruments in order to ensure that all nodes can be calibrated against one of the reference with a path no longer than $k$ hops. Then Maag et al. [45] [61] and Arfire et al. [44] extended this work to models with multiple-kind variables, with and without time dependency. They showed that the complexity of the model should be tuned based on the frequency of rendez-vous.

In a similar way, Markert et al. [17] introduced a calibration strategy based on rendez-vous but with a particular focus on privacy aspects by design for the exchange of data.

Kizel et al. [62] also proposed a multi-hop calibration method that consists into collocating two devices for a certain time, one being the reference to the other, and then moving the freshly calibrated device close to another non-calibrated, a reference instrument being introduced in the loop to reset the error that accumulates. The advantage is that the error is related to the number of hops that took place like in [25].

Sailhan et al. [24] developed a multi-hop, multi-party calibration scheme with the addition of an assessment protocol for the relevancy of the calibration, based on a weighted directed hypergraph of the network, the weights indicating the quality of the calibration. The presented strategy was applied to blind networks but as in [25], it could be extended to partially blind networks. 
Fonollosa et al. [63] used various models for calibration of chemical sensors with an approach called "calibration transfer" which is a kind of multi-hop calibration. Indeed, the principle is to calibrate one of the instruments and then apply the same model on other sensors to calibrate, eventually with a transformation to properly map the measurement spaces. This approach was also used by Laref et al. [64]. Such strategies are widely use in the field of spectroscopy [65]. It however requires that the measuring instruments are more or less behaving the same under identical varying conditions, which is not always the case when dealing with low cost sensors.

\section{E. Blind macro calibration}

Blind calibration strategies for SN were first developed as a way to localize spatially the nodes in a static network. Whitehouse et al. [66] proposed to solve an optimization problem ensuring that consistency and geometrical constraints were respected. Ihler et al. [67] proposed nonparametric belief propagation instead. Taylor et al. [68] developed an inference technique using a Bayesian filter. However, as they targeted spatial localization, most of these methods based their algorithms on electromagnetic or acoustic [69] propagation (time delay, intensity loss...), and cannot be applied directly to other calibration problems.

Bychkovskiy et al. [70] proposed a first solution that could be used for any measurand, provided that the existence of relations between instruments of the network are known. It demands first to estimate the parameters of each existing relationship between instruments in the network. Then, the consistency of the derived relationships must be maximized to be resilient to circular dependencies. This technique was applied to a dense static SN and has not been yet extended to a mobile SN. In theory, it could also be applied to a SN with reference nodes but there are no report on the topic.

Later, Balzano et al. [19] developed a blind and partiallyblind calibration strategy for static SN suitable for any measurands, without any prior knowledge on existing relations between the values of instruments. They tested it notably on temperature, light intensity or $\mathrm{CO}_{2}$ level measurements. The key postulate is that the $\mathrm{SN}$ is dense enough to oversample the signal of interest. They proposed that the true signal lies in a subspace of the space formed by the measured values. Considering the prior choice of the subspace, the parameters of the calibration relationships for all nodes are then estimated using singular value decomposition (SVD) or by solving a system of equations using a least square estimator. This method was extended later in [71] to provide a total least square formulation and also in [72] to take into account outliers and separate them from the measurement matrix.

Alternately, Takruri et al. [73] [74] [75] addressed calibration as a drift compensation problem. They proposed to proceed recursively: measured values at step $n$ are first corrected with predicted drifts obtained at step $n-1$, then the next measurements are predicted using support vector regression (SVR). Finally, the predicted values are used to estimate the drifts using a Kalman filter. Kumar et al. [76] [77] replaced SVR by kriging, which is a method of interpolation originally from geostatistics, as a prediction method for next values.
Ramakrishnan et al. [78] proposed another blind calibration strategy based on a gossip-based distributed consensus strategy, with SAGE algorithm used for parameter estimation. Later Buadhachain et al. [29] used an expectationmaximization algorithm instead. The consensus-based approach is interesting as it can reduce the communication bandwidth.

Bilen et al. [20] extended the problem of blind calibration to the case of sparse input signals, which are measurements with missing/useless information, and exploited compressive sensing to estimate the sensor's corrective gains.

Cambareri et al. [79] proposed a non-convex formulation of the problem and gave a formal criteria of convergence of the calibration method enabling to estimate corrective gains for the values.

For mobile nodes, C. Wang et al. [80] proposed a method which exploits the moments of the measurements, here the average and the variance. They formulate calibration as a optimization problem to minimize the difference between the moments of the true signal and the measured signal. The method was extended in [81] and [26] for different explicit expressions of the calibration relationship. The approach was later tuned by C. Xiang et al. [82] to address specifically mobile crowdsensing, with sensors embedded in mobile phones for instance. Dorffer et al. [83] [84] [34] considered mobile SN calibration with mono-kind variables and linear relationships for spatially sparse signals by using matrix factoring, with an extension regarding non-linear calibration [85].

Wang et al. [21] proposed an extension of [19] relaxing some hypothesis and based their estimation on a Kalman filter with the help of a drift detection strategy. The approach was improved in [86] in terms of drift detection, number of sensors allowed to drift at the same time and pre-processing of the input signal with wavelet denoising. It was then extended for sparse signals with either a Bayesian approach [53] or a deep learning approach [87].

Yang et al. [88] also based their work on the idea of [19]. They prove that, if the underlying signals follow a first-order auto-regressive process, then the parameters of the linear calibration model are recoverable. They use a Bayesian nonparametric model to do so.

Overall, macro calibration methods do not suffer from error propagation issues unlike pairwise approaches. However, because of the absence of references, they usually require large amount of data, which is typically available in the case of high density static networks or mobile networks with high frequency of rendez-vous.

\section{F. Group strategies}

On the first hand, group calibration strategies are used to calibrate multiple instruments located at the same place and measuring different quantities that could influence the calibration relationship. It was shown that their corrected values were more accurate when exploiting multiple-kind variables in calibration relationships [35] [41] [42]. Therefore, strategies [25] that were developed for pairwise and mono-kind variables calibration in the first place were extended to group calibration [44] [45] [61]. New methods were also developed. 


\begin{tabular}{|c|c|c|c|c|c|c|c|c|c|c|c|c|c|}
\hline \multirow[t]{2}{*}{ Papers } & \multicolumn{3}{|c|}{$\begin{array}{l}\text { Availability of } \\
\text { reference } \\
\text { instruments }\end{array}$} & \multicolumn{3}{|c|}{$\begin{array}{l}\text { Instruments } \\
\text { grouping } \\
\text { strategies }\end{array}$} & \multicolumn{4}{|c|}{$\begin{array}{l}\text { Kinds of variables } \\
\text { in calibration } \\
\text { relationships }\end{array}$} & \multicolumn{3}{|c|}{$\begin{array}{l}\text { Mobility of } \\
\text { the } \\
\text { instruments }\end{array}$} \\
\hline & $\mathrm{R}$ & PB & $\mathrm{B}$ & $\mathrm{P}$ & $\mathrm{Gr}$ & Ma & $1 \mathrm{~V}$ & $1 \mathrm{VT}$ & MV & MVT & $\mathrm{M}$ & $\mathrm{S}$ & MS \\
\hline Ramanathan et al. $\mid \overline{54}]$ & $\checkmark$ & - & - & $\checkmark$ & - & - & $\checkmark$ & - & - & - & - & $\checkmark$ & - \\
\hline Miluzzo et al. [27] & $\checkmark$ & - & - & - & $\checkmark$ & - & $\checkmark$ & - & - & - & $\checkmark$ & - & $\checkmark$ \\
\hline$\overline{\text { Deshmukh et al. }}|\overline{89}|$ & $\checkmark$ & - & - & $\checkmark$ & - & - & $\checkmark$ & - & - & - & - & $\checkmark$ & - \\
\hline Spinelle et al. $[41]$ & $\checkmark$ & - & - & $\checkmark$ & $\checkmark$ & - & $\checkmark$ & - & $\checkmark$ & - & - & $\checkmark$ & - \\
\hline Moltchanov et $\overline{\text { al. }} .|\overline{56}|$ & $\checkmark$ & - & - & $\checkmark$ & - & - & $\checkmark$ & - & - & - & - & $\checkmark$ & - \\
\hline Gao et al. & $\checkmark$ & - & - & $\checkmark$ & - & - & $\checkmark$ & - & $\checkmark$ & - & - & $\checkmark$ & - \\
\hline Lin et al. $\mid \overline{55}$ & $\checkmark$ & - & - & $\checkmark$ & - & - & $\checkmark$ & - & - & - & - & $\checkmark$ & - \\
\hline Fang et al. 138 & $\checkmark$ & - & - & - & $\checkmark$ & - & - & - & $\checkmark$ & - & - & - & - \\
\hline Martin et al. $\mid \overline{4} 6]$ & $\checkmark$ & $\checkmark$ & - & - & $\checkmark$ & - & - & - & $\checkmark$ & - & - & $\checkmark$ & - \\
\hline Sun et al. $\mid 43$ & $\checkmark$ & - & - & - & $\checkmark$ & - & - & - & $\checkmark$ & - & - & $\checkmark$ & - \\
\hline Zimmerman et al. $\lceil\overline{91}]$ & $\checkmark$ & - & - & - & $\checkmark$ & - & - & - & $\checkmark$ & - & - & $\checkmark$ & - \\
\hline Tsujita et al. $\mid 58$ & - & $\checkmark$ & $\checkmark$ & $\checkmark$ & - & - & $\checkmark$ & - & - & - & $\checkmark$ & - & $\checkmark$ \\
\hline Tsujita et al. $\mid \overline{57}$ & - & $\checkmark$ & $\checkmark$ & $\checkmark$ & $\checkmark$ & - & - & - & $\checkmark$ & - & - & $\checkmark$ & - \\
\hline Y. Xiang et al. & - & $\checkmark$ & $\checkmark$ & $\checkmark$ & - & - & $\checkmark$ & - & - & - & $\checkmark$ & - & $\checkmark$ \\
\hline Hasenfratz et al. $|\overline{25}|$, Saukh et al. $\mid \overline{33}\rceil|\overline{59}|$ & - & $\checkmark$ & $\checkmark$ & $\checkmark$ & - & - & $\checkmark$ & - & - & - & $\checkmark$ & - & $\checkmark$ \\
\hline Fu et al. $[60]$ & - & $\checkmark$ & $\checkmark$ & $\checkmark$ & - & - & $\checkmark$ & - & - & - & $\checkmark$ & - & $\checkmark$ \\
\hline Maag et al. $\mid 45][\overline{61}$ & - & $\checkmark$ & $\checkmark$ & - & $\checkmark$ & - & - & - & $\checkmark$ & - & $\checkmark$ & - & $\checkmark$ \\
\hline Arfire et al. 44 & - & $\checkmark$ & $\checkmark$ & - & $\checkmark$ & - & $\checkmark$ & $\checkmark$ & $\checkmark$ & $\checkmark$ & $\checkmark$ & - & $\checkmark$ \\
\hline Markert et al. [T] & - & $\checkmark$ & $\checkmark$ & $\checkmark$ & $\checkmark$ & - & $\checkmark$ & - & - & - & $\checkmark$ & - & $\checkmark$ \\
\hline Kizel et al. 62 & - & $\checkmark$ & - & $\checkmark$ & - & - & $\checkmark$ & - & - & - & - & $\checkmark$ & - \\
\hline Sailhan et al. $|\overline{24}|$ & - & - & $\checkmark$ & $\checkmark$ & $\checkmark$ & - & $\checkmark$ & - & - & - & $\checkmark$ & - & - \\
\hline Fonollosa et al. $|\overline{63}|$ & - & $\checkmark$ & $\checkmark$ & $\checkmark$ & - & - & $\checkmark$ & - & - & - & - & $\checkmark$ & - \\
\hline Whitehouse et al. $\mid \overline{66}$ & - & - & $\checkmark$ & - & - & $\checkmark$ & $\checkmark$ & - & - & - & - & $\checkmark$ & - \\
\hline Ihler et al. $\mid 67$ & - & $\checkmark$ & $\checkmark$ & - & $\checkmark$ & - & $\checkmark$ & - & - & - & - & $\checkmark$ & - \\
\hline Taylor et al. $|\overline{68}|$ & - & - & $\checkmark$ & - & - & $\checkmark$ & $\checkmark$ & - & - & - & - & $\checkmark$ & - \\
\hline Tan et al. $\mid 69]$ & - & - & $\checkmark$ & - & $\checkmark$ & - & $\checkmark$ & - & - & - & - & $\checkmark$ & - \\
\hline Bychkovskiy et al. $|70|$ & - & $\checkmark$ & $\checkmark$ & $\checkmark$ & - & - & $\checkmark$ & - & - & - & - & $\checkmark$ & - \\
\hline Balzano et al. $|19|$, Lipor et al. $\mid 71]$, Dorffer et al. $\mid 72$ & - & $\checkmark$ & $\checkmark$ & - & - & $\checkmark$ & $\checkmark$ & - & - & - & - & $\checkmark$ & - \\
\hline Takruri et al. $\mid 73]|\overline{74}| \overline{75}$ & - & - & $\checkmark$ & - & - & $\checkmark$ & $\checkmark$ & - & - & - & - & $\checkmark$ & - \\
\hline Kumar et al. 176 177 & - & - & $\checkmark$ & - & $\checkmark$ & - & $\checkmark$ & - & - & - & - & $\checkmark$ & - \\
\hline Ramakrishnan et al. $|\overline{78}|$ & - & - & $\checkmark$ & - & - & $\checkmark$ & $\checkmark$ & - & - & - & - & $\checkmark$ & - \\
\hline Buadhachain et al. $\mid 29$ & - & - & $\checkmark$ & - & $\checkmark$ & $\checkmark$ & $\checkmark$ & - & - & - & $\checkmark$ & - & $\checkmark$ \\
\hline Bilen et al. $[20]$ & - & - & $\checkmark$ & - & - & $\checkmark$ & $\checkmark$ & - & - & - & - & $\checkmark$ & - \\
\hline Cambareri et al. 179 & - & - & $\checkmark$ & - & - & $\checkmark$ & $\checkmark$ & - & - & - & - & $\checkmark$ & - \\
\hline C. Wang et al. $|\overline{80}| \overline{81}|\overline{26}|$ & - & - & $\checkmark$ & - & - & $\checkmark$ & $\checkmark$ & - & - & - & $\checkmark$ & - & - \\
\hline C. Xiang et al. $|\overline{82}|$ & - & - & $\checkmark$ & - & - & $\checkmark$ & $\checkmark$ & - & - & - & $\checkmark$ & - & - \\
\hline Dorffer et al. $[83]|\overline{85}| \overline{84} \mid \overline{34}]$ & - & $\checkmark$ & $\checkmark$ & - & - & $\checkmark$ & $\checkmark$ & - & - & - & $\checkmark$ & - & - \\
\hline Y. Wang et al. $\mid 21][53]|87| \mathrm{Li} \mid 86]$ & - & - & $\checkmark$ & - & - & $\checkmark$ & - & $\checkmark$ & - & - & - & $\checkmark$ & - \\
\hline Ye et al. $\mid 23]$ & - & - & $\checkmark$ & $\checkmark$ & - & - & $\checkmark$ & - & - & - & $\checkmark$ & - & - \\
\hline De Vito et al. $\mid \overline{22}\lceil\mid 35]$ & - & - & $\checkmark$ & - & $\checkmark$ & - & - & - & $\checkmark$ & - & - & $\checkmark$ & - \\
\hline Son et al. [92], Lee [93] & - & - & $\checkmark$ & - & - & $\checkmark$ & $\checkmark$ & - & - & - & $\checkmark$ & - & - \\
\hline Stankovic et al. $\mid 31] \mid 32]$ & - & - & $\checkmark$ & - & - & $\checkmark$ & $\checkmark$ & - & - & - & - & $\checkmark$ & - \\
\hline Fishbain et al. $\mid \overline{30}$ & - & $\checkmark$ & $\checkmark$ & - & $\checkmark$ & - & $\checkmark$ & - & - & - & - & $\checkmark$ & - \\
\hline Popoola et al. & - & - & $\checkmark$ & $\checkmark$ & - & - & - & - & $\checkmark$ & - & - & $\checkmark$ & - \\
\hline Yan et al. 48 & - & - & $\checkmark$ & - & $\checkmark$ & - & - & - & $\checkmark$ & - & - & $\checkmark$ & - \\
\hline Yang et al. $[\overline{88}$ & - & - & $\checkmark$ & - & - & $\checkmark$ & $\checkmark$ & - & - & - & - & $\checkmark$ & - \\
\hline Mueller et al. $[49]$ & - & - & $\checkmark$ & - & $\checkmark$ & - & - & - & $\checkmark$ & - & - & $\checkmark$ & - \\
\hline Kim et al. $[\overline{9} 4]$ & - & - & $\checkmark$ & - & $\checkmark$ & - & - & - & $\checkmark$ & - & - & $\checkmark$ & - \\
\hline Barcelo-Ordinas et al. $\mid 50$ & - & - & $\checkmark$ & - & $\checkmark$ & - & - & - & $\checkmark$ & - & - & $\checkmark$ & - \\
\hline
\end{tabular}

TABLE I

IN SITU CALIBRATION STRATEGIES FOR SENSOR NETWORKS

R: Reference-Based, PB: Partially blind, B: Blind, P: Pairwise, Gr: Group, Ma: Macro, 1V: Mono-Kind VARiables Without time, 1Vt: MONO-KIND VARIABLES WITH TIME, MV: MULTIPLE-KIND VARIABLES WITHOUT TIME, MVT: MULTIPLE-KIND VARIABLES WITH TIME, M: EXCLUSIVELy MOBILE, S: EXCLUSIVELy STATIC, MS: Mobile AND STATIC

Based on results like [37] [41] [42], Zimmerman et al. [91] introduced a calibration strategy using random forests with multiple-kind variable relationships for measuring systems when the latter are colocated to reference instruments.

Kim et al. [94] recently presented an approach of blind group calibration with prior information on the crosssensitivities of sensors, known from laboratory experiments, in order to build calibration relationships with multiple-kind variables.

On the other hand, other works proposed group calibration strategies in order to reinforce the confidence in the values used as standards.

Miluzzo et al. [27] proposed a calibration technique with multiple reference nodes in a SN by formulating a distributed average consensus problem to estimate the offset of each nonreference node. The concept of sensing factor was introduced. It refers to the area within which the measurand value can be assumed to be identical for the reference instruments and the instruments to calibrate.

Lee et al. [92] [93] proposed a blind group approach 
for mobile nodes. The area of deployment is divided into several non-overlapping regions. It is assumed that calibration relationships exist in each of these area. This is used to formulate the parameter estimation problem as a Laplacian linear equation relating the drift, the measured values and noise.

Stankovic et al. [31] developed a novel methodology based on exploiting the neighbors of each node. Relations in the network are expressed in a matrix and, as there are groups of neighbors, the matrix can be decomposed into blocks. Each block represents a group of relations with parameters to estimate, reducing the problem compared to a macro blind calibration. This approach was extended in [32] to better deal with the case of additive measurement noise.

Fishbain et al. [30] proposed a method of aggregation for non-calibrated SN relying on a group-consensus strategy.

Fang et al. [38] later introduced reference instruments that are measuring different quantities, in view of analyzing the influence quantities in the calibration relationship.

\section{TOWARD PERFORMANCE EVALUATION OF IN SITU CALIBRATION STRATEGIES}

In the preceding review, no comparison of strategies in terms of performance was made. However, each publication on a novel strategy proposes a validation step either based on simulations [34], laboratory or field experiments [56] or both [19].

It is achieved in the following manner: reference values, which are values that should have been measured by healthy instruments, are compared to corrected values after the application of the strategy to evaluate.

Reference values are obtained by simulation or from reference instruments in the field. Then a dataset of measured values is created, either by extraction from the reference dataset (with the addition of faults to the sensors data, in an approach through numerical simulation) or using actual sensors deployed in the field. Afterwards, The calibration strategy is applied on this measured dataset, and the corrected dataset is compared to the reference dataset using appropriate metrics.

Regarding approaches by simulation, the reference values are computed numerically. Different protocols have been proposed to generate them. It can be based on 2D Gaussian fields [83] or ARMA processes [87] for instance. Disturbances like noise or drifts are added to the reference values in order to generate the measured values. Concerning laboratory or field experiments, reference values are usually obtained with high accuracy instruments and measured values from low cost sensors. There are lots of datasets produced and used in various studies (for instance a deployment at James Reserve used in [19], the OpenSense Zurich Dataset [95]), though the resulting data often become unavailable over time.

Various metrics are then used to assess the performance of the strategies such as root-mean-square error, Pearson's coefficient, metrics developed in works on the evaluation of the performance of sensors [96] or ones related to official recommendations such as the data quality objective (DQO) in the context of air quality measuring instruments [97] [14] [98]. They are mostly based on prior information concerning the expected corrected values.

In general, there appears to be a strong need for systematic tools and protocols [99] [14] enabling to compare across studies the performance of in situ calibration methodologies. Indeed, results can be compared even if they were not obtained on the same test case as it was done in [100], but to have the most significance, they should be obtained on the same experiment. Unlike the common practice in the field of machine learning [101] and even if datasets are disclosed or simulation protocols are presented in detail, there is no report of a test case widely used across the literature. The comparison between methods is also limited by code availability: while a few authors [71] [72] have shared their code, in most cases existing strategies must be fully reimplemented for comparison purposes, as in [21]. This is time-consuming and drives the authors to only focus on comparisons between strategies with very specific characteristics, for instance those which use machine learning techniques [102] [103] [104].

Moreover, the availability of tools for comparisons across studies would be highly useful to enable end-users to select the most appropriate method for a given use case. So far, only a few methods provide formal criteria regarding to the suitability of a method to a given configuration such as in [34] or [19]. In addition, it would also help to configure the strategies in an optimal manner, for instance regarding the periodicity of calibrations e.g., according to a defined rule-every night in [56]-or upon decision by an expert user.

Throughout the literature, it appears that the quality of input data impacts the performance of the calibration. Outliers contained in datasets have a more important influence on the calibration results than the overall data accuracy [38]. As an early work on the topic, Ramanathan et al. [54] observed that, even with individual field calibration for each sensor performed with a mobile chemistry lab at the time of deployment, the resulting data was corrupted with anomalous entries. Thus, through simulation with controlled injection of outliers, their influence on the calibration results could be quantified and strategies to tolerate or remove them could be designed.

To quantify data quality, a few contributions on in situ calibration now offer indicators concerning this topic: Ganeriwal et al. [105] proposed a reputation-based framework in order to validate the integrity of data based on a Bayesian formulation and in which nodes computes metrics regarding past and future behaviors of the others. Yim et al. [106] developed a malicious node detection system based on variation tests and confidence level evaluation using a weighted majority voting system. Hasenfratz et al. [107] presented a model-based approach to determine accuracy bounds of noisy sensor values for sensor networks. Radanovic et al. [108] also introduced a reputation system and particularly focus on malicious aspects with the design of an influence limiter independent of the reporting strategy of the malicious nodes. Le Taro et al. [109] proposed in a simple way the comparison of a confidence indicator between nodes, related to the duration elapsed since their last calibration with another device. This topic is of major interest 
to provide more trust into measured values [110]

\section{CONCLUSION}

Instrumenting territories to monitor environmental phenomena may require the use of hundreds or thousands of measuring instruments. This is becoming possible nowadays thanks to the emergence of low-cost sensors. Among the multiple challenges that must be tackled regarding these technologies, evaluation of the quality of their calibration, and its correction if needed, is critical to ensure that measurement results remain accurate over time. In situ calibration is a response to the issue of calibrating dense sensor networks at a reasonable cost. To achieve that, the use of the properties of the sensor networks have a key role in the strategies. We propose in this paper a classification of such methodologies applied to environmental sensors, based on four groups of categories capturing both network architecture and algorithmic principles: the availability of reference instruments in the network, the mobility of the instruments, the kind of input variables in the calibration relationships, and the instruments grouping strategy (pairwise, macro or by group) used for a calibration procedure.

Our review shows that relatively few papers address methodological issues related to reference-based pairwise strategies, as this approach is the closest to a "traditional" calibration approach and features relatively little challenges. Partially blind and blind pairwise calibration methods, which often focus on mobile nodes, are more complex as they require to define calibration relationships between non-reference nodes, which translates into error propagation issues. Macro calibration approaches are mostly used to deal with reference-less situation. Their challenge lies in defining valid calibration relationships between non-reference sensors. Group strategies appear to improve on performance of both pairwise and macro strategies with or without reference instruments. Most methods are based on calibration relationships with mono-kind variables and a linear expression, but more complex models are progressively appearing to better address the complexity of environmental sensing. Likewise, while most work initially focused on static networks, there is now a strong interest for mobile nodes as they allow for physical rendez-vous between nodes, which reduces the impact of the physical variability of the phenomena between static distant nodes.

Though each method has its own performance indicators, it is observed that there is no commonly used test case enabling a standardized comparison between methods. The development of both systematic tools for comparison of performance between methods and indicators on data and calibration quality, could strongly foster the applicability of in situ calibration on actual deployment and help to build trust in environmental sensor data from low-cost sensors.

\section{REFERENCES}

[1] J. K. Hart and K. Martinez, "Environmental Sensor Networks: A revolution in the earth system science?" Earth-Science Reviews, vol. 78, no. 3-4, pp. 177-191, 2006.
[2] A. Boubrima, W. Bechkit, H. Rivano, and L. Soulhac, "Leveraging the Potential of WSN for an Efficient Correction of Air Pollution Fine-Grained Simulations," in The 27th International Conference on Computer Communications and Networks (ICCCN 2018), Hangzou, China, 2018, pp. 1-9. [Online]. Available: https://hal.inria.fr/hal-01781389/

[3] A. Marjovi, A. Arfire, and A. Martinoli, "High Resolution Air Pollution Maps in Urban Environments Using Mobile Sensor Networks," 2015 International Conference on Distributed Computing in Sensor Systems, pp. 11-20, 2015. [Online]. Available: http: //ieeexplore.ieee.org/document/7165019/

[4] P. Kumar, L. Morawska, C. Martani, G. Biskos, M. Neophytou, S. Di Sabatino, M. Bell, L. Norford, and R. Britter, "The rise of low-cost sensing for managing air pollution in cities," Environment International, vol. 75, pp. 199-205, 2015. [Online]. Available: http://dx.doi.org/10.1016/j.envint.2014.11.019

[5] P. W. Rundel, E. A. Graham, M. F. Allen, J. C. Fisher, and T. C. Harmon, "Environmental sensor networks in ecological research," New Phytologist, vol. 182, no. 3, pp. 589-607, 2009.

[6] W. Y. Yi, K. M. Lo, T. Mak, K. S. Leung, Y. Leung, and M. L. Meng, "A Survey of Wireless Sensor Network Based Air Pollution Monitoring Systems," Sensors, vol. 15, no. 12, pp. 31392-31 427, 2015.

[7] X. Fang and I. Bate, "Issues of using wireless sensor network to monitor urban air quality," in International Workshop on the Engineering of Reliable, Robust, and Secure Embedded Wireless Sensing Systems (FAILSAFE). ACM., 2017.

[8] L. Morawska, P. K. Thai, X. Liu, A. Asumadu-sakyi, G. Ayoko, A. Bartonova, A. Bedini, F. Chai, B. Christensen, M. Dunbabin, J. Gao, G. S. W. Hagler, R. Jayaratne, P. Kumar, A. K. H. Lau, P. K. K. Louie, M. Mazaheri, Z. Ning, N. Motta, B. Mullins, M. Rahman, Z. Ristovski, M. Sha, D. Tjondronegoro, D. Westerdahl, and R. Williams, "Applications of low-cost sensing technologies for air quality monitoring and exposure assessment : How far have they gone ?" Environment International, vol. 116, pp. 286-299, 2018.

[9] A. L. Clements, W. G. Griswold, A. Rs, J. E. Johnston, M. M. Herting, J. Thorson, A. Collier-oxandale, and M. Hannigan, "Low-Cost Air Quality Monitoring Tools : From Research to Practice (A Workshop Summary)," Sensors, vol. 17, no. 11, 2017. [Online]. Available: http://www.mdpi.com/1424-8220/17/11/2478

[10] BIPM, IEC, IFCC, ILAC, IUPAC, IUPAP, ISO, and OIML, International vocabulary of metrology - Basic and general concepts and associated terms (VIM), 3rd ed. JCGM 200:2012, 2012. [Online]. Available: http://www.bipm.org/vim

[11] M. Kamionka, P. Breuil, and C. Pijolat, "Calibration of a multivariate gas sensing device for atmospheric pollution measurement," Sensors and Actuators, B: Chemical, vol. 118, no. 1-2, pp. 323-327, 2006.

[12] D. M. Broday, A. Arpaci, A. Bartonova, N. Castell-Balaguer, T. ColeHunter, F. R. Dauge, B. Fishbain, R. L. Jones, K. Galea, M. JovaševićStojanović, D. Kocman, T. Martinez-Iñiguez, M. Nieuwenhuijsen, J. A. Robinson, V. Svecova, and P. Thai, "Wireless distributed environmental sensor networks for air pollution measurement-the promise and the current reality," Sensors, vol. 17, no. 10, 2017.

[13] Measurement specialities. (2012) Hs1101lf relative humidity sensor datasheet. [Online]. Available: https://docs-emea.rs-online.com/ webdocs/142c/0900766b8142cdcd.pdf

[14] B. Maag, Z. Zhou, and L. Thiele, "A Survey on Sensor Calibration in Air Pollution Monitoring Deployments," IEEE Internet of Things Journal, vol. 5, no. 6, pp. 4857-4870, 2018.

[15] J.-C. Laprie, "Dependable Computing and Fault-Tolerance : Concepts and Terminology," in Proceedings of International Symposium on Fault-Tolerant Computing, vol. III, 1985, pp. 2-11.

[16] A. Avižienis, J.-C. Laprie, B. Randell, and C. Landwehr, "Basic Concepts and Taxonomy of Dependable and Secure Computing," IEEE Transactions on Dependable and Secure Computing, vol. 1, no. 1, pp. 11-33, 2004

[17] J.-F. Markert, M. Budde, G. Schindler, M. Klug, and M. Beigl, "Privacy-Preserving Collaborative Blind Macro-Calibration of Environmental Sensors in Participatory Sensing," EAI Endorsed Transactions on Internet of Things, vol. 18, no. 10, pp. 1-8, 2018.

[18] A. Vergara, S. Vembu, T. Ayhan, M. A. Ryan, M. L. Homer, and R. Huerta, "Chemical gas sensor drift compensation using classifier ensembles," Sensors and Actuators, B: Chemical, vol. 166-167, pp. 320-329, 2012. [Online]. Available: http://dx.doi.org/10.1016/j.snb. 2012.01.074

[19] L. Balzano and R. Nowak, "Blind Calibration of Sensor Networks,' 2007 6th International Symposium on Information Processing in Sensor Networks, pp. 79-88, 2007. 
[20] C. Bilen, G. Puy, R. Gribonval, and L. Daudet, "Convex Optimization Approaches for Blind Sensor Calibration using Sparsity," IEEE Transactions on Signal Processing, pp. 1-11, 2013. [Online] Available: http://dx.doi.org/10.1109/TSP.2014.2342651

[21] Y. Wang, A. Yang, Z. Li, P. Wang, and H. Yang, "Blind drift calibration of sensor networks using signal space projection and Kalman filter," in 2015 IEEE Tenth International Conference on Intelligent Sensors, Sensor Networks and Information Processing (ISSNIP), 2015, pp. 1-6.

22] S. De Vito, P. Delli Veneri, E. Esposito, M. Salvato, W. Bright, R. L. Jones, and O. A. Popoola, "Dynamic Multivariate Regression For OnField Calibration Of High Speed Air Quality Chemical Multi-Sensor Systems," in 2015 XVIII AISEM Annual Conference, 2015, pp. 1-3.

[23] H. Ye, T. Gu, X. Tao, and J. Lu, "SBC : Scalable Smartphone Barometer Calibration through Crowdsourcing," in 11th International Conference on Mobile and Ubiquitous Systems: Computing, Networking and Services, 2014.

[24] F. Sailhan, V. Issarny, and O. Tavares-Nascimiento, "Opportunistic Multiparty Calibration for Robust Participatory Sensing," in Proceedings - 14th IEEE International Conference on Mobile Ad Hoc and Sensor Systems, MASS 2017, 2017, pp. 435-443.

[25] D. Hasenfratz, O. Saukh, and L. Thiele, "On-the-fly calibration of lowcost gas sensors," in Proceedings of the 9th European Conference on Wireless Sensor Networks, G. P. Picco,, and W. Heinzelman, Eds. Springer Berlin Heidelberg, 2012, pp. 228-244.

[26] C. Wang, P. Ramanathan, and K. K. Saluja, "Blindly Calibrating Mobile Sensors Using Piecewise Linear Functions," in 2009 6th Annual IEEE Communications Society Conference on Sensor, Mesh and Ad Hoc Communications and Networks, 2009, pp. 1-9.

[27] E. Miluzzo, N. D. Lane, A. T. Campbell, and R. Olfati-Saber, "CaliBree: A self-calibration system for mobile sensor networks," in Distributed Computing in Sensor Systems, 2008, pp. 314-331.

[28] Y. Xiang, L. Bai, R. Piedrahita, R. P. Dick, Q. Lv, M. Hannigan, and L. Shang, "Collaborative calibration and sensor placement for mobile sensor networks," in 2012 ACM/IEEE 11th International Conference on Information Processing in Sensor Networks (IPSN), 2012, pp. 73-83. [Online]. Available: http://dl.acm.org/citation.cfm? doid=2185677.2185687

[29] S. Buadhachain and G. Provan, "A model-based control method for decentralized calibration of wireless sensor networks," in 2013 American Control Conference, 2013, pp. 6571-6576.

[30] B. Fishbain and E. Moreno-Centeno, "Self Calibrated Wireless Distributed Environmental Sensory Networks," Scientific Reports, vol. 6, pp. 1-10, 2016. [Online]. Available: http://dx.doi.org/10.1038/ srep24382

[31] M. S. Stankovic, S. S. Stankovic, and K. H. Johansson, "Distributed Blind Calibration in Lossy Sensor Networks via Output Synchronization," IEEE Transactions on Automatic Control, vol. 60 , no. 12 , pp. 3257-3262, 2015. [Online]. Available: http://ieeexplore.ieee.org/document/7094268/

[32] "Asynchronous Distributed Blind Calibration of Sensor Networks under Noisy Measurements," IEEE Transactions on Control of Network Systems, vol. 5, no. 1, pp. 571-582, 2018. [Online]. Available: http://ieeexplore.iee.org/document/7762879/

[33] O. Saukh, D. Hasenfratz, C. Walser, and L. Thiele, "On rendezvous in mobile sensing networks," in In Springer RealWSN, 2013, pp. 29-42.

[34] C. Dorffer, M. Puigt, G. Delmaire, and G. Roussel, "Informed Nonnegative Matrix Factorization Methods for Mobile Sensor Network Calibration," IEEE Transactions on Signal and Information Processing over Networks, vol. 4, no. 4, pp. 667-682, 2018.

[35] E. Esposito, S. De Vito, M. Salvato, V. Bright, R. L. Jones, and O. Popoola, "Dynamic neural network architectures for on field stochastic calibration of indicative low cost air quality sensing systems," Sensors and Actuators, B: Chemical, vol. 231, pp. 701-713, 2016

[36] J. H. Sohn, M. Atzeni, L. Zeller, and G. Pioggia, "Characterisation of humidity dependence of a metal oxide semiconductor sensor array using partial least squares," Sensors and Actuators, B: Chemical, vol. 131 , no. 1 , pp. 230-235, 2008.

[37] W. Jiao, G. Hagler, R. Williams, R. Sharpe, R. Brown, D. Garver, R. Judge, M. Caudill, J. Rickard, M. Davis, L. Weinstock, S. Zimmerdauphinee, and K. Buckley, "Community Air Sensor Network (CAIRSENSE) project : evaluation of low-cost sensor performance in a suburban environment in the southeastern United States," Atmospheric Measurement Techniques, vol. 9, pp. 5281-5292, 2016

[38] X. Fang and I. Bate, "Using Multi-parameters for Calibration of Lowcost Sensors in Urban Environment," in Proceedings of the 2017 In- ternational Conference on Embedded Wireless Systems and Networks, 2017, pp. 1-11.

[39] R. Jayaratne, X. Liu, P. Thai, M. Dunbabin, and L. Morawska, "The influence of humidity on the performance of a low-cost air particle mass sensor and the effect of atmospheric fog," Atmospheric Measurement Techniques, vol. 11, no. 8, pp. 4883-4890, 2018

[40] P. Wei, Z. Ning, S. Ye, L. Sun, F. Yang, K. C. Wong, D. Westerdahl, and P. K. Louie, "Impact analysis of temperature and humidity condition on electrochemical sensor response in ambient air quality monitoring,' Sensors, vol. 18, no. 2, 2018.

[41] L. Spinelle, M. Gerboles, M. G. Villani, M. Aleixandre, and F. Bonavitacola, "Field calibration of a cluster of low-cost available sensors for air quality monitoring. Part A: Ozone and nitrogen dioxide," Sensors and Actuators, B: Chemical, vol. 215, pp. 249-257, 2015. [Online]. Available: http://dx.doi.org/10.1016/j.snb.2015.03.031

[42] — " "Field calibration of a cluster of low-cost commercially available sensors for air quality monitoring. Part $\mathrm{B}$ : $\mathrm{NO}, \mathrm{CO}$ and CO2," Sensors and Actuators, B: Chemical, vol. 238, pp. 706-715, 2017. [Online]. Available: http://dx.doi.org/10.1016/j.snb.2016.07.036

[43] L. Sun, D. Westerdahl, and Z. Ning, "Development and Evaluation of A Novel and Cost-Effective Approach for Low-Cost NO2 Sensor Drift Correction," Sensors, vol. 17, no. 8, 2017. [Online]. Available: http://www.mdpi.com/1424-8220/17/8/1916

[44] A. Arfire, A. Marjovi, and A. Martinoli, "Model-based rendezvous calibration of mobile sensor networks for monitoring air quality," in 2015 IEEE SENSORS, 2015, pp. 1-4.

[45] B. Maag, O. Saukh, D. Hasenfratz, and L. Thiele, "Pre-Deployment Testing, Augmentation and Calibration of Cross-Sensitive Sensors," in Proceedings of the 2016 International Conference on Embedded Wireless Systems and Networks, 2016, pp. 169-180. [Online]. Available: http://dl.acm.org/citation.cfm?id=2893711.2893735

[46] C. R. Martin, N. Zeng, A. Karion, R. R. Dickerson, X. Ren, B. N. Turpie, and K. J. Weber, "Evaluation and environmental correction of ambient $\mathrm{CO} 2$ measurements from a low-cost NDIR sensor," At mospheric Measurement Techniques, vol. 10, no. 7, pp. 2383-2395, 2017

[47] O. A. Popoola, G. B. Stewart, M. I. Mead, and R. L. Jones, "Development of a baseline-temperature correction methodology for electrochemical sensors and its implications for long-term stability," Atmospheric Environment, vol. 147, pp. 330-343, 2016. [Online]. Available: http://dx.doi.org/10.1016/j.atmosenv.2016.10.024

[48] K. Yan and D. Zhang, "Calibration transfer and drift compensation of e-noses via coupled task learning," Sensors and Actuators: B. Chemical, vol. 225, pp. 288-297, 2016. [Online]. Available: http://dx.doi.org/10.1016/j.snb.2015.11.058

[49] M. Mueller, J. Meyer, and C. Hueglin, "Design of an ozone and nitrogen dioxide sensor unit and its long-term operation within a sensor network in the city of Zurich," Atmospheric Measurement Techniques, vol. 10 , no. 10 , pp. 3783-3799, 2017.

[50] J. M. Barcelo-Ordinas, J. Garcia-Vidal, M. Doudou, S. Rodrigo-Munoz, and A. Cerezo-Llavero, "Calibrating low-cost air quality sensors using multiple arrays of sensors," in IEEE Wireless Communications and Networking Conference, WCNC, 2018, pp. 1-6.

[51] D. M. Holstius, A. Pillarisetti, K. R. Smith, and E. Seto, "Field calibrations of a low-cost aerosol sensor at a regulatory monitoring site in California," Atmospheric Measurement Techniques, vol. 7, no. 4, pp. 1121-1131, 2014

[52] M. Bruins, J. Willem, W. W. J. V. D. Sande, A. V. Belkum, and A. Bos, "Enabling a transferable calibration model for metal-oxide type electronic noses," Sensors and Actuators: B. Chemical, vol. 188, pp. 1187-1195, 2013. [Online]. Available: http://dx.doi.org/10.1016/j.snb.2013.08.006

[53] Y. Wang, A. Yang, Z. Li, X. Chen, P. Wang, and H. Yang, "Blind drift calibration of sensor networks using sparse Bayesian learning," IEEE Sensors Journal, vol. 16, no. 16, pp. 6249-6260, 2016.

[54] N. Ramanathan, L. Balzano, M. Burt, D. Estrin, T. C. Harmon, C. Harvey, J. Jay, E. Kohler, S. Rothenberg, and M. Srivastava, "Rapid Deployment with Confidence: Calibration and Fault Detection in Environmental Sensor Networks," Tech. Rep., 2006. [Online] Available: https://escholarship.org/uc/item/8v26b5qh

[55] C. Lin, N. Masey, H. Wu, M. Jackson, D. J. Carruthers, S. Reis, R. M. Doherty, I. J. Beverland, and M. R. Heal, "Practical Field Calibration of Portable Monitors for Mobile Measurements of Multiple Air Pollutants," Atmosphere, vol. 8, no. 12, 2017.

[56] S. Moltchanov, I. Levy, Y. Etzion, U. Lerner, D. M. Broday, and B. Fishbain, "On the feasibility of measuring urban air pollution by wireless distributed sensor networks," Science of the Total 
Environment, vol. 502, pp. 537-547, 2015. [Online]. Available: http://dx.doi.org/10.1016/j.scitotenv.2014.09.059

[57] W. Tsujita, A. Yoshino, H. Ishida, and T. Moriizumi, "Gas sensor network for air-pollution monitoring," Sensors and Actuators, B: Chemical, vol. 110, no. 2, pp. 304-311, 2005.

[58] W. Tsujita, H. Ishida, and T. Moriizumi, "Dynamic gas sensor network for air pollution monitoring and its auto-calibration," in SENSORS, 2004 IEEE, Vienna, 2004, pp. 56-59. [Online]. Available: https://ieeexplore.ieee.org/document/1426098/

[59] O. Saukh, D. Hasenfratz, and L. Thiele, "Reducing multi-hop calibration errors in large-scale mobile sensor networks," in Proceedings of the 14th International Conference on Information Processing in Sensor Networks, 2015, pp. 274-285. [Online]. Available: http://doi.acm.org/10.1145/2737095.2737113

[60] K. Fu, W. Ren, and W. Dong, "Multihop Calibration for Mobile Sensing : k-hop Calibratability and Reference Sensor Deployment," in IEEE INFOCOM 2017 - IEEE Conference on Computer Communications, 2017, pp. 1-9.

[61] B. Maag, Z. Zhou, O. Saukh, and L. Thiele, "SCAN: Multi-Hop Calibration for Mobile Sensor Arrays," in Proc. ACM Interact. Mob. Wearable Ubiquitous Technol., vol. 1, no. 2, 2017, pp. 19:1-19:21. [Online]. Available: http://doi.acm.org/10.1145/3090084

[62] F. Kizel, Y. Etzion, R. Shafran-Nathan, I. Levy, B. Fishbain, A. Bartonova, and D. M. Broday, "Node-to-node field calibration of wireless distributed air pollution sensor network," Environmental Pollution, vol. 233, pp. 900-909, 2018. [Online]. Available: http://www.sciencedirect.com/science/article/pii/S0269749117311818

[63] J. Fonollosa, L. Fernandez, A. Gutierrez-Galvez, R. Huerta, and S. Marco, "Calibration transfer and drift counteraction in chemical sensor arrays using Direct Standardization," Sensors and Actuators, B: Chemical, vol. 236, pp. 1044-1053, 2016.

[64] R. Laref, E. Losson, A. Sava, and M. Siadat, "Support vector machine regression for calibration transfer between electronic noses dedicated to air pollution monitoring," Sensors, vol. 18, no. 11, 2018. [Online]. Available: http://www.mdpi.com/1424-8220/18/11/3716

[65] J. J. Workman Jr, "A Review of Calibration Transfer Practices and Instrument Differences in Spectroscopy," Applied Spectroscopy, vol. 72 , no. 3 , pp. $340-365,2018$.

[66] K. Whitehouse and D. Culler, "Calibration as parameter estimation in sensor networks," in Proceedings of the 1st ACM International Workshop on Wireless Sensor Networks and Applications. ACM, 2002, pp. 59-67. [Online]. Available: http://doi.acm.org/10.1145/ 570738.570747

[67] A. T. Ihler, J. W. Fisher III, R. L. Moses, and A. S. Willsky, "Nonparametric belief propagation for self-calibration in sensor networks," in Third International Symposium on Information Processing in Sensor Networks, 2004. IPSN 2004, 2004, pp. 225-233.

[68] C. Taylor, A. Rahimi, J. Bachrach, H. Shrobe, and A. Grue, "Simultaneous localization, calibration, and tracking in an ad hoc sensor network," in 2006 5th International Conference on Information Processing in Sensor Networks, 2006, pp. 27-33.

[69] R. Tan, G. Xing, Z. Yuan, X. Liu, and J. Yao, "System-level Calibration for Data Fusion in Wireless Sensor Networks," ACM Transactions on Sensor Networks, vol. 9, no. 3, pp. 28:1-28:27, 2013.

[70] V. Bychkovskiy, S. Megerian, D. Estrin, and M. Potkonjak, "A collaborative approach to in-place sensor calibration," in Proceedings of the 2nd International Conference on Information Processing in Sensor Networks, 2003, pp. 301-316.

[71] J. Lipor and L. Balzano, "Robust blind calibration via total leas squares," in 2014 IEEE International Conference on Acoustics, Speech and Signal Processing (ICASSP), 2014, pp. 4244-4248.

[72] C. Dorffer, M. Puigt, G. Delmaire, and G. Roussel, "Outlier-robust calibration method for sensor networks," in Proceedings of the 2017 IEEE International Workshop of Electronics, Control, Measurement, Signals and their Application to Mechatronics, ECMSM 2017, 2017.

[73] M. Takruri and S. Challa, "Drift aware wireless sensor networks," in 2007 10th International Conference on Information Fusion, 2007, pp. $1-7$.

[74] M. Takruri, K. Aboura, and S. Challa, "Distributed recursive algorithm for auto calibration in drift aware wireless sensor networks," Innovations and Advanced Techniques in Systems, Computing Sciences and Software Engineering, pp. 21-25, 2008.

[75] M. Takruri, S. Rajasegarar, S. Challa, C. Leckie, and M. Palaniswami, "Online Drift Correction in Wireless Sensor Networks Using SpatioTemporal Modeling," in 2008 11th International Conference on Information Fusion, 2008, pp. 1-8.
[76] D. Kumar, S. Rajasegarar, and M. Palaniswami, "Automatic sensor drift detection and correction using spatial kriging and kalman filtering," in 2013 IEEE International Conference on Distributed Computing in Sensor Systems, 2013, pp. 183-190.

[77] —_ "Geospatial Estimation-Based Auto Drift Correction in Wireless Sensor Networks," ACM Transactions on Sensor Networks, vol. 11, no. 3, pp. 50:1-50:39, 2015

[78] N. Ramakrishnan, E. Ertin, and R. L. Moses, "Distributed signature learning and calibration for large-scale sensor networks," in 2010 Conference Record of the Forty Fourth Asilomar Conference on Signals, Systems and Computers, 2010, pp. 1545-1549.

[79] V. Cambareri and L. Jacques, "A Greedy Blind Calibration Method for Compressed Sensing with Unknown Sensor Gains," in 2017 IEEE International Symposium on Information Theory (ISIT), 2017, pp. 1132-1136. [Online]. Available: http://arxiv.org/abs/1610.02851

[80] C. Wang, P. Ramanathan, and K. K. Saluja, "Moments based blind calibration in mobile sensor networks," in 2008 IEEE International Conference on Communications, 2008, pp. 896-900.

[81] _ _ "Calibrating nonlinear mobile sensors," in 2008 5th Annual IEEE Communications Society Conference on Sensor, Mesh and Ad Hoc Communications and Networks, 2008, pp. 533-541.

[82] C. Xiang and P. Yang, "An Iterative Method of Sensor Calibration in Participatory Sensing Network," in 2013 IEEE 10th International Conference on Mobile Ad-Hoc and Sensor Systems, 2013, pp. 431-432. [Online]. Available: http://ieeexplore.ieee.org/document/6680278/

[83] C. Dorffer, M. Puigt, G. Delmaire, and G. Roussel, "Blind Calibration of Mobile Sensors Using Informed Nonnegative Matrix Factorization," in 12th International Conference on Latent Variable Analysis and Signal Separation (LVA/ICA 2015), 2015, pp. 497-505.

[84] _ "Blind mobile sensor calibration using an informed nonnegative matrix factorization with a relaxed rendezvous model," in 2016 IEEE International Conference on Acoustics, Speech and Signal Processing (ICASSP), 2016, pp. 2941-2945.

[85] — "Nonlinear mobile sensor calibration using informed seminonnegative matrix factorization with a Vandermonde factor," in 2016 IEEE Sensor Array and Multichannel Signal Processing Workshop (SAM), 2016

[86] Z. Li, Y. Wang, A. Yang, and H. Yang, "Drift detection and calibration of sensor networks," in 2015 International Conference on Wireless Communications and Signal Processing, WCSP 2015, 2015, pp. 1-6.

[87] Y. Wang, A. Yang, X. Chen, P. Wang, Y. Wang, and H. Yang, "A Deep Learning Approach for Blind Drift Calibration of Sensor Networks," IEEE Sensors Journal, vol. 17, no. 13, pp. 4158-4171, 2017.

[88] J. Yang, W. P. Tay, and X. Zhong, "A dynamic Bayesian nonparametric model for blind calibration of sensor networks," in 2017 IEEE International Conference on Acoustics, Speech and Signal Processing (ICASSP), 2017, pp. 4207-4211. [Online]. Available: http://ieeexplore.ieee.org/document/7952949/

[89] S. Deshmukh, K. Kamde, A. Jana, S. Korde, R. Bandyopadhyay, R. Sankar, N. Bhattacharyya, and R. A. Pandey, "Calibration transfer between electronic nose systems for rapid In situ measurement of pulp and paper industry emissions," Analytica Chimica Acta, vol. 841, pp. 58-67, 2014. [Online]. Available: http://dx.doi.org/10.1016/j.aca. 2014.05 .054

[90] M. Gao, J. Cao, and E. Seto, "A distributed network of low-cost continuous reading sensors to measure spatiotemporal variations of PM2.5 in Xi'an, China," Environmental Pollution, vol. 199, pp. 56-65, 2015. [Online]. Available: http://dx.doi.org/10.1016/j.envpol.2015.01. 013

[91] N. Zimmerman, A. A. Presto, S. P. Kumar, J. Gu, A. Hauryliuk, E. S. Robinson, A. L. Robinson, and R. Subramanian, "A machine learning calibration model using random forests to improve sensor performance for lower-cost air quality monitoring," Atmospheric Measurement Techniques, vol. 11, no. 1, pp. 291-313, 2018

[92] S.-c. Son and B.-t. Lee, "A Blind Calibration Scheme using a Graph Model for Optical Mobile Sensor Network," in 2014 12th International Conference on Optical Internet 2014 (COIN), no. 3, 2014, pp. 5-6.

[93] B. T. Lee, S. C. Son, and K. Kang, "A blind calibration scheme exploiting mutual calibration relationships for a dense mobile sensor network," IEEE Sensors Journal, vol. 14, no. 5, pp. 1518-1526, 2014.

[94] J. Kim, A. A. Shusterman, K. J. Lieschke, C. Newman, and R. C. Cohen, "The Berkeley Atmospheric CO2 Observation Network: Field calibration and evaluation of low-cost air quality sensors," Atmospheric Measurement Techniques, vol. 11, no. 4, pp. 1937-1946, 2018.

[95] J. J. Li, B. Faltings, O. Saukh, D. Hasenfratz, and J. Beutel, "Sensing the Air We Breathe-The OpenSense Zurich Dataset." in Proceedings of the Twenty-Sixth AAAI Conference on Artificial Intelligence, 2012, 
pp. 323-325. [Online]. Available: http://dl.acm.org/citation.cfm?id= 2900728.2900775

[96] B. Fishbain, U. Lerner, N. Castell, T. Cole-Hunter, O. A. Popoola, D. M. Broday, T. Martinez-Iñiguez, M. Nieuwenhuijsen, M. JovaševićStojanović, D. Topalović, R. L. Jones, K. S. Gaela, Y. Etzion, F. Kizel, Y. N. Golumbic, A. Baram-Tsabari, T. Yacobi, D. Drahler, J. A. Robinson, D. Kocman, M. Horvat, V. Svecova, A. Arpaci, and A. Bartonova, "An evaluation tool kit of air quality micro-sensing units," Science of the Total Environment, vol. 575, pp. 639-648, 2017. [Online]. Available: http://dx.doi.org/10.1016/j.scitotenv.2016.09.061

[97] N. Castell, F. R. Dauge, P. Schneider, M. Vogt, U. Lerner, B. Fishbain, D. M. Broday, and A. Bartonova, "Can commercial low-cost sensor platforms contribute to air quality monitoring and exposure estimates?" Environment International, vol. 99, pp. 293-302, 2017. [Online]. Available: http://dx.doi.org/10.1016/j.envint.2016.12.007

[98] C. Borrego, J. Ginja, M. Coutinho, C. Ribeiro, K. Karatzas, T. Sioumis, N. Katsifarakis, K. Konstantinidis, S. De Vito, E. Esposito, M. Salvato, P. Smith, N. André, P. Gérard, L. A. Francis, N. Castell, P. Schneider, M. Viana, M. C. Minguillón, W. Reimringer, R. P. Otjes, O. von Sicard, R. Pohle, B. Elen, D. Suriano, V. Pfister, M. Prato, S. Dipinto, and M. Penza, "Assessment of air quality microsensors versus reference methods: The EuNetAir Joint Exercise Part II," Atmospheric Environment, vol. 193, pp. 127-142, 2018. [Online]. Available: https://doi.org/10.1016/j.atmosenv.2018.08.028

[99] A. C. Lewis, E. Von Schneidemesser, and R. E. Peltier, "Low-cost sensors for the measurement of atmospheric composition: overview of topic and future applications," Tech. Rep. May, 2018.

[100] E. S. Cross, L. R. Williams, D. K. Lewis, G. R. Magoon, T. B. Onasch, M. L. Kaminsky, D. R. Worsnop, and J. T. Jayne, "Use of electrochemical sensors for measurement of air pollution: Correcting interference response and validating measurements," Atmospheric Measurement Techniques, vol. 10, no. 9, pp. 3575-3588, 2017

[101] D. Dheeru and E. Karra Taniskidou, "UCI machine learning repository," 2017. [Online]. Available: http://archive.ics.uci.edu/ml

[102] E. Esposito, S. De Vito, M. Salvato, G. Fattoruso, and G. Di Francia, "Computational Intelligence for Smart Air Quality Monitors Calibration," in Computational Science and Its Applications - ICCSA 2017, 2017, pp. 443-454

[103] E. Esposito, S. De Vito, M. Salvato, G. Fattoruso, V. Bright, R. L. Jones, D.-f.-d. E. Fermi, and P. Na, "Stochastic Comparison of Machine Learning Approaches to Calibration of Mobile Air Quality Monitors," in Sensors: Proceedings of the Third National Conference on Sensors, 2018.

[104] J. G. Casey and M. P. Hannigan, "Testing the performance of field calibration techniques for low-cost gas sensors in new deployment locations: across a county line and across Colorado," Atmospheric Measurement Techniques, vol. 11, no. 11, pp. 6351-6378, 2018 [Online]. Available: https://www.atmos-meas-tech.net/11/6351/2018/

[105] S. Ganeriwal, L. K. Balzano, and M. B. Srivastava, "Reputation-based Framework for High Integrity Sensor Networks," ACM Transactions on Sensor Networks, vol. 4, no. 3, 2008.

[106] S.-j. Yim and Y.-h. Choi, "Neighbor-Based Malicious Node Detection in Wireless Sensor Networks," Wireless Sensor Network, vol. 4, no. 9 , pp. 219-225, 2012

[107] D. Hasenfratz, O. Saukh, and L. Thiele, "Model-Driven Accuracy Bounds for Noisy Sensor Readings," in 2013 IEEE International Conference on Distributed Computing in Sensor Systems, 2013, pp. $165-174$.

[108] G. Radanovic and B. Faltings, "Limiting the Influence of Low Quality Information in Community Sensing," in Proceedings of the 2016 International Conference on Autonomous Agents and Multiagent Systems, 2016, pp. 873-881.

[109] L. Le Taro and H. Rivano, "Simulating Collaborative Sensor Calibration: Convergence and Cost," in SENSORCOMM 2017 - 11th International Conference on Sensor Technologies and Applications, 2017, pp. 1-5. [Online]. Available: https://hal.inria.fr/hal-01654371

[110] J. Lopez, R. Roman, I. Agudo, and C. Fernandez-gago, "Trust Management Systems for Wireless Sensor Networks : Best Practices," Computer Communications, vol. 33, no. 9, pp. 1086-1093, 2010.

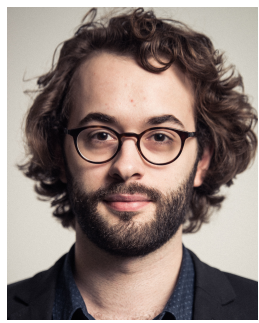

Florentin Delaine is a $\mathrm{Ph} . \mathrm{D}$. student at Efficacity, IFSTTAR and École Polytechnique since 2017. He graduated from École Normale Supérieure ParisSaclay, France, in 2017 from which he obtained a first M.S. in Applied Physics and a second M.S. in Design and Control of Critical Systems. His research interests include data science for sensor networks with a focus on metrology and dependability for these systems.

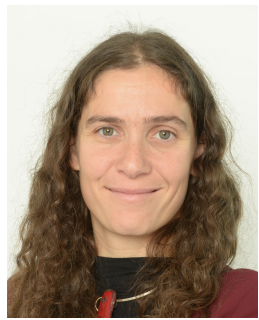

Bérengère Lebental graduated from Ecole Polytechnique's (Paris Saclay University, France) Engineering Program in 2006 and both its Physics and its Nanotechnology Master of Science Programs in 2007. She obtained her Ph.D. in Civil Engineering from Université Paris-Est, France, in 2010. Since 2010, she is research scientist at Laboratory of Physics of Interfaces and Thin Films (École Polytechnique, CNRS) and French Institute of Science and Technology for Transport, Development and Network (IFSTTAR). Her research focuses on nanoenabled sensors for smart city applications, including design, fabrication, reliability analysis, IoT integration, field deployment and data exploitation. She has coordinated several large scale national and EU research projects on sensors for smart cities, such as Sense-City (http://sense-city.ifsttar.fr/ or Proteus (http://www.proteus-sensor.eu/). She is cofounder of the startup Altaroad for connected roads.

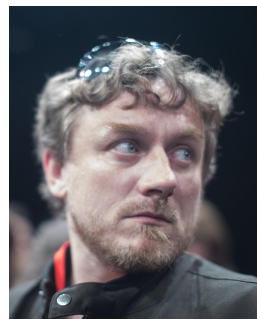

Hervé Rivano is University Professor at INSA Lyon. He is the head of the Inria/INSA Lyon common team Agora of the CITI lab. The team focuses on wireless networks for digital cities. He obtained his $\mathrm{PhD}$ in november 2003 from the University of Nice-Sophia Antipolis and graduated from the École Normale Supérieure de Lyon. His research interests include combinatorial optimization applied to network design and provisioning. He focuses on capacity/energy tradeoff for urban cellular and mesh networks design and low cost and dense wireless sensor networks for environmental sensing. 